\title{
New Betulinic Acid Derivatives as Potent Proteasome Inhibitors
}

\author{
Keduo Qian ${ }^{a,}{ }^{,}$, Sang-Yong Kim ${ }^{\mathrm{a}}$, Hsin-Yi Hung ${ }^{\mathrm{a}}$, Li Huang ${ }^{\mathrm{b}}$, Chin-Ho Chen ${ }^{\mathrm{b}}$, and Kuo- \\ Hsiung Lee ${ }^{a, c,{ }^{*}}$ \\ aNatural Products Research Laboratories, UNC Eshelman School of Pharmacy, University of \\ North Carolina, Chapel Hill, NC 27599, USA \\ bDepartment of Surgery, Duke University Medical Center, Durham, NC 27710, USA \\ ${ }^{\circ}$ Chinese Medicine Research and Development Center, China Medical University and Hospital, \\ Taichung 401, Taiwan
}

\begin{abstract}
In this study, 22 new betulinic acid (BA) derivatives were synthesized and tested for their inhibition of the chymotrypsin-like activity of $20 \mathrm{~S}$ proteasome. From the SAR study, we concluded that the $\mathrm{C}-3$ and $\mathrm{C}-30$ positions are the pharmacophores for increasing the proteasome inhibition effects, and larger lipophilic or aromatic side chains are favored at these positions. Among the BA derivatives tested, compounds 13, 20, and $\mathbf{2 1}$ showed the best proteasome inhibition activity with $\mathrm{IC}_{50}$ values of $1.42,1.56$, and $1.80 \mu \mathrm{M}$, respectively, which are three- to four-fold more potent than the proteasome inhibition controls LLM-F and lactacystin.
\end{abstract}

Betulinic acid (BA, 33-hydroxy-lup-20(29)-en-28-oic acid, 1) is a pentacyclic triterpene present in many plant species, e.g., Triphyophyllum peltatum, Ancistrocladus heyneanus, Ziziphi fructus, Diospyros leucomelas, Tetracera boliviana and Syzygium formosanum, ${ }^{1-3}$ and can be obtained in quantity from the bark of the London plane tree, Platanus acerifolia. ${ }^{4-6}$ BA (1) exhibits diverse biological activities including anti-retroviral, antibacterial, anti-malarial, anti-inflammatory, anti-cancer, anti-oxidant, and anthelmintic properties. Our previous research on 1 resulted in the discovery of bevirimat [DSB, 3-O-(3', 3'-dimethylsuccinyl)-betulinic acid, 2] (Figure 1), ${ }^{7}$ which exhibits remarkable anti-HIV-1 activity with a mean $\mathrm{EC}_{50}$ value of $<10 \mathrm{nM}$ against primary and drug-resistant HIV-1 isolates, ${ }^{7,8}$ representing a unique first in a class of anti-HIV compounds termed maturation inhibitors (MIs). ${ }^{8,} 9$ Bevirimat has recently succeeded in Phase IIb clinical trials. ${ }^{6,10-12}$ In the further evaluation of $\mathbf{2}$, we discovered that HIV-1 virions produced in the presence of $\mathbf{2}$ contained higher amounts of the cellular protein APOBEC 3G, which is usually degraded by cellular proteasomes. We then postulated that the elevated APOBEC3G level in these viral infected cells may be due to the inhibition of proteasomes by $\mathbf{2}$.

Proteasomes are responsible for more than $80 \%$ of intracellular protein degradation. ${ }^{13}$ The most common form of the proteasome is known as $26 \mathrm{~S}$ proteasome, which contains one $20 \mathrm{~S}$ core particle structure and two 19S regulatory caps. All 20S particles consist of four stacked heptameric ring structures that are themselves composed of two different types of subunits,

(C) 2011 Elsevier Ltd. All rights reserved.

*To whom correspondence should be addressed. K. Qian: Phone: 919-966-1394, kdqian@ unc.edu; K.-H. Lee: Phone: 919-962-0066, Fax: 919-966-3893, khlee@unc.edu.

Publisher's Disclaimer: This is a PDF file of an unedited manuscript that has been accepted for publication. As a service to our customers we are providing this early version of the manuscript. The manuscript will undergo copyediting, typesetting, and review of the resulting proof before it is published in its final citable form. Please note that during the production process errors may be discovered which could affect the content, and all legal disclaimers that apply to the journal pertain. 
$\alpha$ and $\beta$. Alpha subunits are structural in nature, whereas $\beta$ subunits are predominantly catalytic. The $\beta$ subunits of proteasomes have three major proteolytic activities:

chymotrypsin-like ( $\beta 5)$, trypsin-like ( $\beta 2)$, and caspase-like ( $\beta 1)$. These proteolytic activities enable the proteasome to cleave proteins into small peptides. Proteasomes are involved in many essential cellular functions, such as cell cycle regulation, cell differentiation, signal transduction pathways, antigen processing for appropriate immune responses, stress signaling, inflammatory responses, and apoptosis. Targeting the proteasome-ubiquitin pathway has thus been considered a novel strategy for the treatment of various disorders such as neurodegenerative diseases, cancers, and inflammatory diseases. ${ }^{14,15}$

In our initial screening, we found that $\mathbf{2}$ inhibited the chymotrypsin-like activity of $20 \mathrm{~S}$ proteasome with an $\mathrm{IC}_{50}$ value of $9.2 \mu \mathrm{M}$, but it did not inhibit the caspase-like activity, and had a very weak inhibitory effect $\left(\mathrm{IC}_{50}: 26.29 \mu \mathrm{M}\right)$ on the trypsin-like activity. ${ }^{16}$ These results are favorable because normal cells may suffer fewer consequences from treatment with $\mathbf{2}$, as two of the main proteolytic activities of proteasomes are retained. Indeed, the anticancer drug bortezomib (PS341), the first successful proteasome inhibitor for the treatment of multiple myeloma, also showed differential inhibition of the three catalytic activities of proteasomes, which is believed to be critical for its clinical benefit in disease treatment. ${ }^{14,17,18}$ In addition, compound 2 did not inhibit the cellular proteases cathepsin B (cysteine protease) and DPPIV (DPP4, CD26, serine protease) in our screening. ${ }^{16}$ Consequently, it may have reduced side effects compared with other proteasome inhibitors such as peptide aldehydes and peptide boronates, which also inhibit these cellular proteases. Therefore, to better establish a structure-activity relationship (SAR) of the proteasome regulatory activity of BA derivatives, diverse substituted BA analogs were designed. This paper reports herein the synthesis and proteasome inhibitory activity of these BA derivatives.

Because $\mathbf{2}$ is a C-3 modified BA derivative, we first synthesized eleven 3-O-acyl-BA analogs (3-13) to investigate the impacts of different C-3 modifications on the proteasome inhibitory activity of BA derivatives. As shown in Scheme 1, 3,4,5-trimethoxybenzoyl chloride was reacted with 1 in the presence of $\mathrm{Et}_{3} \mathrm{~N}$ in $\mathrm{CH}_{2} \mathrm{Cl}_{2}$ at room temperature to yield compound 3. Diverse carboxylic acids were first treated with EDCI and DMAP in $\mathrm{CH}_{2} \mathrm{Cl}_{2}$ for $1 \mathrm{~h}$, before compound $\mathbf{1}$ and $\mathrm{Et}_{3} \mathrm{~N}$ were added into the system. The mixtures were stirred at room temperature to yield compounds 4-10 and 13. 1,2,3,6-Tetrahydrophthalic and diglycolic anhydrides were refluxed with $\mathbf{1}$ in the presence of DMAP in pyridine to provide compounds 11 and 12, respectively. To investigate the impact of 28- and 30-subsitutions on BA's proteasome inhibitory effects, nine 28,30-disubstituted BA analogs (14-22) were synthesized by the method reported previously (Scheme 2$)^{19}$ and were also tested against $20 \mathrm{~S}$ proteasome. Briefly, BA was first protected with acetic anhydride to yield $\mathbf{2 5}$, which was treated with oxalyl chloride and then reacted with leucine methyl ester to furnish $\mathbf{2 6}$. Allylic bromination of 26 was carried out using NBS in dilute acetonitrile to provide 27. Saponification of 27 yielded 14. Meanwhile, nucleophilic displacement of the bromide of 27 was conducted by treating varied nucleophilic compounds with $\mathrm{NaH}$, followed by their reaction with 27 in a microwave apparatus at $120^{\circ} \mathrm{C}$. Saponification of the resulting mixtures in $\mathrm{MeOH}-\mathrm{H}_{2} \mathrm{O}$ converted the intermediate esters to carboxylic acids to yield the target compounds 15-22. Two compounds with only a C-28 side chain, LH15bh (23) and IC9564 (24) (Figure 1), ${ }^{20,21}$ were also tested for their proteasome inhibition activity to investigate the influence of $\mathrm{C}-28$ mono-substitution.

Table 1 lists the results for inhibition of the chymotrypsin-like activity of 20S proteasome shown by BA derivatives 3-24 ${ }^{22}$ together with compound $\mathbf{2}$. Known proteasome inhibitors Ac-Leu-Leu-Met-CHO (LLM-F) and lactacystin were also included in the in vitro $20 \mathrm{~S}$ proteasome assay ${ }^{23}$ as controls. From the results with compounds $\mathbf{2 - 1 3}$, we can conclude 
that addition of a C-3 ester group to $\mathbf{1}$ can confer proteasome inhibition activity and the identity of the ester group can influence the potency. Comparing the activity of 3-10, we found that compound $\mathbf{6}$ with an unsubstituted benzoate ester side chain and compound $\mathbf{5}$ with 2',4',5'-trimethoxy substituted benzoate ester side chain at the C-3 position showed moderate inhibition activity ( $\mathrm{IC}_{50}: 11.06$ and $10.91 \mu \mathrm{M}$, respectively) on the chymotrypsinlike activity of $20 \mathrm{~S}$ proteasome. Compounds $\mathbf{3}, \mathbf{4}$, and $\mathbf{7}$ without ortho-methoxy substitution on benzoate ester side chain abolished or greatly decreased the proteasome inhibition activity. In a comparison of three compounds with different brominated benzoate side chains, bromide on the 2'- or 3'-position (8 and 10) was advantageous ( $\mathrm{IC}_{50}: 7.99$ and 7.05 $\mu \mathrm{M}$, respectively), while bromide on the 4'-position (9) was detrimental. Interestingly, while compound 12, with a polar oxygen atom in the middle of a diglycolic ester side chain was inactive, compounds with both a cyclohexene ring (11) and a phenyl ring (13), located centrally within a dicarboxylic side chain were significantly active. Compound 11, with a 1,2,3,6-tetrahydrophthlate ester side chain, exhibited increased proteasome inhibition activity with an $\mathrm{IC}_{50}$ value of $2.96 \mu \mathrm{M}$. Elongation of the C-3 side chain to $3^{\prime}-$ acetylphenylacetate (13) further increased the proteasome inhibition activity to an $\mathrm{IC}_{50}$ value of $1.42 \mu \mathrm{M}$, which is six-fold better than 2 and four-fold better than LLM-F and lactacystin. Among the synthesized 3-O-acyl BA analogs, compound $\mathbf{1 3}$ showed the best activity, suggesting that an aromatic lipophilic ester side chain with a carboxylic acid terminus at the $\mathrm{C}-3$ position can significantly increase the proteasome inhibition activity of BA derivatives.

With respect to the effects of 28- and 30-substitutions on the proteasome activity, we found that all nine 28,30-disubstituted BA analogs (14-22) showed inhibitory activity towards the chymotrypsin-like activity of $20 \mathrm{~S}$ proteasome. However, the 28 -substituted BA derivatives 23 and 24 did not inhibit the 20 S proteasome to any degree. We therefore concluded that the proteasome inhibition activity of the 28,30-disubstituted BA compounds comes mainly from the contribution of the $\mathrm{C}-30$ substituents. Within the $\mathrm{C}-30$ side chains, larger ether substituents (16-22) were favored compared with bromide (14) or a smaller ethoxy group (15). Among 16-22, the rank order of potency based on the C-30 ether substituent was 4'bromophenethoxy $(\mathbf{2 0})=4^{\prime}$-chlorophenethoxy $(\mathbf{2 1})>4^{\prime}$-fluorophenethoxy $(\mathbf{1 9})=$ phenethoxy $(\mathbf{1 7})>$ propoxy $(\mathbf{1 6})=4^{\prime}$-methyoxyphenethoxy $(\mathbf{1 8})=2^{\prime}$-morpholinoethoxy (22). Overall, compound $\mathbf{1 5}$ with an ethoxy substituent showed the lowest activity among the 28,30-disubstituted BAs with an $\mathrm{IC}_{50}$ value of $13.21 \mu \mathrm{M}$, while compounds $\mathbf{2 0}$ and $\mathbf{2 1}$ with 4'-bromophenethoxy and 4'-chlorophenethoxy substituents, respectively, showed the highest activity with $\mathrm{IC}_{50}$ values of 1.56 and $1.80 \mu \mathrm{M}$, respectively, three-fold better than LLM-F and lactacystin. Thus, longer C-30 side chains with aromatic substitutions were favored for the proteasome inhibition activity.

In summary, this study showed that substituted BA analogs can be proteasome inhibitors, and the $\mathrm{C}-3$ and $\mathrm{C}-30$ positions of $\mathrm{BA}$ are the pharmacophores for improving the proteasome inhibition activity. Within the C-3 and C-30 substitutions, larger side chains with lipophilic or aromatic side chains are favored for increased inhibition of the chymotrypsin-like activity of $20 \mathrm{~S}$ proteasome. C-3 and C-30 modified BA compounds showed low toxicity in our previous anti-AIDS studies, and bevirimat (2), a C-3 substituted BA, has already succeeded in phase IIb clinical trials. Therefore, development of C-3 and/or C-30 modified BA derivatives as proteasome inhibitors might be able to provide new therapeutic agents for treating cancers and inflammatory diseases.

\section{Acknowledgments}

This investigation was supported by Grant GM-084337 from the National Instituted of General Medical Sciences (NIGMS) awarded to C.H.C and AI-077417 from the National Institute of Allergy and Infectious Diseases (NIAID) 
awarded to K.H.L. This study was also supported in part by Taiwan Department of Health Clinical Trial and Research Center of Excellence (DOH100-TD-B-111-004). Efficient purification of all the synthetic BA analogs was performed with a Grace Reveleris $®$ flash chromatography system equipped with RevealX(TM) detection allowing for multisignal (UV/ELSD) collection to low mg quantities. Reveleris ${ }^{\circledR}$ Navigator method optimizer and Grace Reveleris ${ }^{\circledR}$ flash silica cartridges were employed for high quality separations.

\section{References and notes}

1. Chang C-W, Wu T-S, Hsieh Y-S, Kuo S-C, Chao P-DL. J. Nat. Prod. 1999; 62:327. [PubMed: 10075776]

2. Bringmann G, Saeb W, Assi LA, Francois G, Narayanan ASS, Peters K, Peters EM. Planta Med. 1997; 63:255. [PubMed: 9225608]

3. Bae KH, Lee SM, Lee ES, Lee JS, Kang JS. Yakhak Hoechi. 1996; 40:558.

4. Krasutsky PA, Carlson RM, Nesterenko VV, Kolomitsyn IV, Edwardson CF. Application: WO2001010885. 2001

5. Birgit, DGT.; Neubert, R.; Wohlrab, W. US Patent. 2001.

6. Qian, K.; Nitz, TJ.; Yu, D.; Allaway, GP.; Morris-Natschke, SL.; Lee, KH. Natural Product Chemistry for Drug Discovery, for the Royal Society of Chemistry Biomolecular Sciences Series. Vol. 13. Merlion Pharmaceuticals Pte Ltd Press; 2009. p. 374

7. Kashiwada Y, Hashimoto F, Cosentino LM, Chen CH, Garrett PE, Lee KH. J. Med. Chem. 1996; 39:1016. [PubMed: 8676334]

8. Kanamoto T, Kashiwada Y, Kanbara K, Gotoh K, Yoshimori M, Goto T, Sano K, Nakashima H. Antimicrob. Agents Chemother. 2001; 45:1225. [PubMed: 11257038]

9. Li F, Goila-Gaur R, Salzwedel K, Kilgore NR, Reddick M, Matallana C, Castillo A, Zoumplis D, Martin DE, Orenstein JM, Allaway GP, Freed EO, Wild CT. Proc. Natl. Acad. Sci. USA. 2003; 100:13555. [PubMed: 14573704]

10. Smith PF, Ogundele A, Forrest A, Wilton J, Salzwedel K, Doto J, Allaway GP, Martin DE. Antimicrob. Agents Chemother. 2007; 51:3574. [PubMed: 17638699]

11. Martin DE, Blum R, Doto J, Galbraith H, Ballow C. Clin. Pharmacokinet. 2007; 46:589. [PubMed: 17596104]

12. Martin DE, Blum R, Wilton J, Doto J, Galbraith H, Burgess GL, Smith PC, Ballow C. Antimicrob. Agents Chemother. 2007; 51:3063. [PubMed: 17576843]

13. Nencioni A, Grunebach F, Patrone F, Ballestrero A, Brossart P. Leukemia. 2007; 21:30. [PubMed: 17096016]

14. Huang L, Yu D, Ho P, Qian K, Lee KH, Chen CH. Bioorg. Med. Chem. 2008; 16:6696. [PubMed: 18562200]

15. Nalepa G, Rolfe M, Harper JW. Nat. Rev. Drug Discov. 2006; 5:596. [PubMed: 16816840]

16. Huang L, Ho P, Chen CH. FEBS Lett. 2007; 581:4955. [PubMed: 17904555]

17. Kane RC, Bross PF, Farrell AT, Pazdur R. Oncologist. 2003; 8:508. [PubMed: 14657528]

18. Kisselev AF, Callard A, Goldberg AL. J. Biol. Chem. 2006; 281:8582. [PubMed: 16455650]

19. Qian K, Yu D, Chen CH, Huang L, Morris-Natschke SL, Nitz TJ, Salzwedel K, Reddick M, Allaway GP, Lee KH. J. Med. Chem. 2009; 52:3248. [PubMed: 19388685]

20. Sun IC, Chen CH, Kashiwada Y, Wu JH, Wang HK, Lee KH. J. Med. Chem. 2002; 45:4271. [PubMed: 12213068]

21. Huang L, Yuan X, Aiken C, Chen CH. Antimicrobial Agents Chemother. 2004; 48:663.

22. Materials and methods: ${ }^{1} \mathrm{H}$ NMR spectra were measured on a 300 or $400 \mathrm{MHz}$ Varian Gemini 2000 spectrometer using TMS as internal standard. The solvent used was $\mathrm{CDCl}_{3}$. Mass spectra were measured on a Shimadzu LC-MS2010 instrument. Thin-layer chromatography (TLC) was performed on precoated silica gel GF plates purchased from Merck, Inc. All other chemicals were obtained from Aldrich, Inc. Fast purification of crude reaction mixtures was performed on the Grace Reveleris ${ }^{\circledR}$ flash system by prescreening the crude mixtures on TLC plates to select appropriate solvent pairs for purification. Method parameters were automatically loaded when the Reveleris ${ }^{\circledR}$ cartridge was inserted or when the Reveleris ${ }^{\circledR}$ Navigator method optimizer was employed to predict high purity or fastest separations, but were adjusted manually as desired. 
Crude samples were dissolved in the appropriate solvent before being injected automatically via solid loading. Spectroscopic data for target compounds are shown as follows. Compound $3:{ }^{1} \mathrm{H}$ NMR (400 MHz, $\mathrm{CDCl}_{3}$ ): $\delta$ 7.29, 7.27 (1H each, s, Ar-H), 4.76, 4.64 (1H each, s, H-29), 4.60 $(1 \mathrm{H}, \mathrm{m}, \mathrm{H}-3), 3.94\left(3 \mathrm{H}, \mathrm{s}, \mathrm{Ar}-\mathrm{OCH}_{3}\right), 3.91\left(6 \mathrm{H}, \mathrm{s}, 2 \times \mathrm{Ar}_{-} \mathrm{OCH}_{3}\right), 3.06(1 \mathrm{H}, \mathrm{m}, H-19), 1.71(3 \mathrm{H}, \mathrm{s}$, $H$-30), 0.97, 0.90, 0.83, 0.76, 0.70 (3H each, s, $\left.5 \times \mathrm{CH}_{3}\right)$, MS m/z $649.50\left(\mathrm{M}^{-}-1\right)$; Compound 4: ${ }^{1} \mathrm{H}$ NMR (400 MHz, CDCl 3 ): $\delta 7.64\left(1 \mathrm{H}, \mathrm{d}, J=8.0 \mathrm{~Hz}, \mathrm{Ar}-H-6^{\prime}\right), 7.45\left(1 \mathrm{H}, \mathrm{s}, \mathrm{Ar}-H-2^{\prime}\right), 6.84$ $\left(1 \mathrm{H}, \mathrm{d}, J=8.0 \mathrm{~Hz}, \mathrm{Ar}-H-5\right.$ '), $6.03\left(2 \mathrm{H}, \mathrm{s},-\mathrm{OCH}_{2} \mathrm{O}-\right), 4.73,4.60(1 \mathrm{H}$ each, s, $H-29), 4.65(1 \mathrm{H}, \mathrm{m}$, $H-3), 3.10(1 \mathrm{H}, \mathrm{m}, H-19), 1.70(3 \mathrm{H}, \mathrm{s}, H-30), 0.99,0.97,0.96,0.90,0.89$ (3H each, s, $\left.5 \times \mathrm{CH}_{3}\right)$, MS m/z $603.40\left(\mathrm{M}^{-}\right.$- 1); Compound 5: ${ }^{1} \mathrm{H}$ NMR (400 MHz, $\left.\mathrm{CDCl}_{3}\right): \delta 7.44\left(1 \mathrm{H}, \mathrm{s}, \mathrm{Ar}-\mathrm{H}-6^{\prime}\right), 6.52$ $\left(1 \mathrm{H}, \mathrm{s}, \mathrm{Ar}-H-3^{\prime}\right), 4.75,4.62$ (1H each, s, $\left.H-29\right), 4.69(1 \mathrm{H}, \mathrm{dd}, J=8.0,4.0 \mathrm{~Hz}, H-3), 3.93,3.88$, $3.86\left(3 \mathrm{H}\right.$ each, s, $\left.3 \times \mathrm{Ar}^{-O C H} \mathrm{H}_{3}\right), 3.01(1 \mathrm{H}, \mathrm{m}, H-19), 1.70(3 \mathrm{H}, \mathrm{s}, H-30), 1.00,0.97,0.96,0.95$, 0.89 ( $3 \mathrm{H}$ each, s, $\left.5 \times \mathrm{CH}_{3}\right)$, MS m/z $649.70\left(\mathrm{M}^{-}-1\right)$; Compound $6:{ }^{1} \mathrm{H} \mathrm{NMR}\left(400 \mathrm{MHz}, \mathrm{CDCl}_{3}\right)$ : $\delta 8.04\left(2 \mathrm{H}, \mathrm{d}, J=8.0 \mathrm{~Hz}, \mathrm{Ar}-H-2^{\prime}, 6^{\prime}\right), 7.55\left(1 \mathrm{H}, \mathrm{m}, \mathrm{Ar}-H-4^{\prime}\right), 7.45\left(1 \mathrm{H}, \mathrm{m}, \mathrm{Ar}-H-3^{\prime}, 5^{\prime}\right), 4.75,4.62$ (1H each, s, $H-29), 4.71(1 \mathrm{H}, \mathrm{m}, H-3), 3.02(1 \mathrm{H}, \mathrm{m}, H-19), 1.71(3 \mathrm{H}, \mathrm{s}, H-30), 1.00,1.00,0.96$, 0.93, $0.91\left(3 \mathrm{H}\right.$ each, s, $\left.5 \times \mathrm{CH}_{3}\right)$, MS m/z $559.45\left(\mathrm{M}^{-}-1\right)$; Compound 7: ${ }^{1} \mathrm{H} \mathrm{NMR}(400 \mathrm{MHz}$, $\left.\mathrm{CDCl}_{3}\right): \delta 7.68\left(1 \mathrm{H}, \mathrm{d}, J=4.0 \mathrm{~Hz}, \mathrm{Ar}-H-6^{\prime}\right), 7.56\left(1 \mathrm{H}, \mathrm{s}, \mathrm{Ar}-H-2^{\prime}\right), 6.88(1 \mathrm{H}, \mathrm{d}, J=4.0 \mathrm{~Hz}, \mathrm{Ar}-$ $\left.H-5^{\prime}\right), 4.75,4.62$ (1H each, s, $\left.H-29\right), 4.69(1 \mathrm{H}, \mathrm{m}, H-3), 3.96,3.93$ (3H each, s, $2 \times \mathrm{Ar}_{-} \mathrm{OCH}_{3}$ ), $3.02(1 \mathrm{H}, \mathrm{m}, H-19), 1.71(3 \mathrm{H}, \mathrm{s}, H-30), 1.00,0.99,0.96,0.92,0.90\left(3 \mathrm{H}\right.$ each, s, $\left.5 \times \mathrm{CH}_{3}\right), \mathrm{MS} \mathrm{m} / \mathrm{z}$ $619.45\left(\mathrm{M}^{-}-1\right)$; Compound 8: ${ }^{1} \mathrm{H}$ NMR $\left(400 \mathrm{MHz}, \mathrm{CDCl}_{3}\right)$ : $\delta 7.75\left(1 \mathrm{H}, \mathrm{d}, J=8.0 \mathrm{~Hz}, \mathrm{Ar}-\mathrm{H}-6^{\prime}\right)$, $7.64\left(1 \mathrm{H}, \mathrm{d}, J=8.0 \mathrm{~Hz}, \mathrm{Ar}-H-3^{\prime}\right), 7.37-7.28\left(2 \mathrm{H}, \mathrm{m}, \mathrm{Ar}-H-4^{\prime}, 5^{\prime}\right), 4.78(1 \mathrm{H}, \mathrm{m}, H-3), 4.75,4.62$ (1H each, s, $H-29), 3.01(1 \mathrm{H}, \mathrm{m}, H-19), 1.71(3 \mathrm{H}, \mathrm{s}, H-30), 1.00,0.97,0.95,0.94,0.89$ (3H each, s, $\left.5 \times \mathrm{CH}_{3}\right)$, MS m/z $639.40\left(\mathrm{M}^{+}+1\right)$; Compound 9: ${ }^{1} \mathrm{H}$ NMR $\left(400 \mathrm{MHz}, \mathrm{CDCl}_{3}\right): \delta 7.89(2 \mathrm{H}, \mathrm{d}$, $\left.J=8.0 \mathrm{~Hz}, \mathrm{Ar}-H-2^{\prime}, 6^{\prime}\right), 7.59\left(2 \mathrm{H}, \mathrm{d}, J=8.0 \mathrm{~Hz}, \mathrm{Ar}-H-3^{\prime}, 5^{\prime}\right), 4.73,4.60$ (1H each, s, $\left.H-29\right), 4.70$ (1H, m, H-3), 3.02 (1H, m, H-19), 1.70 (3H, s, H-30), 1.00, 0.99, 0.97, 0.91, 0.90 (3H each, s, $5 \times$ $\left.\mathrm{CH}_{3}\right)$, MS m/z $637.65\left(\mathrm{M}^{-}\right.$- 1); Compound 10: ${ }^{1} \mathrm{H} \mathrm{NMR}\left(400 \mathrm{MHz}, \mathrm{CDCl}_{3}\right): \delta 8.15(1 \mathrm{H}, \mathrm{s}, \mathrm{Ar}-$ $\left.H-2^{\prime}\right), 7.95\left(1 \mathrm{H}, \mathrm{d}, J=8.0 \mathrm{~Hz}, \mathrm{Ar}-H-6^{\prime}\right), 7.67\left(1 \mathrm{H}, \mathrm{d}, J=8.0 \mathrm{~Hz}, \mathrm{Ar}-H-4^{\prime}\right), 7.31\left(1 \mathrm{H}, \mathrm{m}, \mathrm{Ar}-H-5^{\prime}\right)$, 4.75, 4.62 (1H each, s, $H-29), 4.71(1 \mathrm{H}, \mathrm{m}, H-3), 3.01(1 \mathrm{H}, \mathrm{m}, H-19), 1.71(3 \mathrm{H}, \mathrm{s}, H-30), 1.00$, 0.99, 0.96, 0.92, 0.91 (3H each, s, $\left.5 \times \mathrm{CH}_{3}\right)$, MS m/z $637.50\left(\mathrm{M}^{-}-1\right)$; Compound 11: ${ }^{1} \mathrm{H}$ NMR (300 MHz, $\left.\mathrm{CDCl}_{3}\right): \delta 5.70\left(2 \mathrm{H}, \mathrm{m}, H-5^{\prime}, 6^{\prime}\right), 4.72,4.60(1 \mathrm{H}$ each, s, $H-29), 4.48(1 \mathrm{H}, \mathrm{m}, H-3)$, $3.02(1 \mathrm{H}, \mathrm{m}, H-19), 2.83,2.72$ (1H each, $\left.\mathrm{m}, H-2^{\prime}, 3^{\prime}\right), 2.32-2.27$ (4H, m, $\left.2 \times \mathrm{CH}_{2}, H_{-1} 4^{\prime}, 7^{\prime}\right), 1.68$ ( $3 \mathrm{H}, \mathrm{s}, \mathrm{H}-30), 1.01,0.97$ ( $3 \mathrm{H}$ each, s, $\left.2 \times \mathrm{CH}_{3}\right), 0.89,0.83,0.81\left(3 \mathrm{H}\right.$ each, s, $\left.3 \times \mathrm{CH}_{3}\right), \mathrm{MS} \mathrm{m} / \mathrm{z}$ $607.40\left(\mathrm{M}^{-}-1\right)$; Compound 12: ${ }^{1} \mathrm{H}$ NMR $\left(300 \mathrm{MHz}, \mathrm{CDCl}_{3}\right)$ : $\delta 4.72,4.60$ (1H each, s, $\left.H-29\right)$, $4.46(1 \mathrm{H}, \mathrm{m}, H-3), 4.23,4.21$ (2H each, s, $\left.H-2^{\prime}, 4^{\prime}\right), 3.01(1 \mathrm{H}, \mathrm{m}, H-19), 1.71(3 \mathrm{H}, \mathrm{s}, H-30), 1.00$, 0.98, 0. 79, 0.88, 0.87 (3H each, s, $\left.5 \times \mathrm{CH}_{3}\right)$, MS $m / z, 571.60\left(\mathrm{M}^{-}-1\right)$; Compound 13: ${ }^{1} \mathrm{H} \mathrm{NMR}$ $\left(400 \mathrm{MHz}, \mathrm{CDCl}_{3}\right): \delta 7.82\left(1 \mathrm{H}, \mathrm{s}, \mathrm{Ar}-H-2^{\prime}\right), 7.57-7.50\left(2 \mathrm{H}, \mathrm{m}, \mathrm{Ar}-H-4^{\prime}, 6^{\prime}\right), 7.30\left(1 \mathrm{H}, \mathrm{m}, \mathrm{Ar}-\mathrm{H}-5^{\prime}\right)$, 4.72, 4.60 (1H each, s, $H-29), 4.40(1 \mathrm{H}, \mathrm{m}, \mathrm{H}-3), 3.63,3.60$ (2H each, s, $\left.2 \times \mathrm{Ar}-\mathrm{CH}_{2}-\mathrm{CO}-\right), 2.98$ (1H, m, $H-19), 1.68(3 \mathrm{H}, \mathrm{s}, H-30), 0.95,0.90,0.82,0.72,0.68\left(3 \mathrm{H}\right.$ each, $\left.\mathrm{s}, 5 \times \mathrm{CH}_{3}\right), \mathrm{MS} \mathrm{m} / \mathrm{z}$ $631.78\left(\mathrm{M}^{-}-1\right)$; Compound 14: ${ }^{1} \mathrm{H}$ NMR $\left(300 \mathrm{MHz}, \mathrm{CDCl}_{3}\right): \delta 5.86(1 \mathrm{H}, \mathrm{d}, J=8.0 \mathrm{~Hz},-$ CONH-), 5.11, 5.02 (1H each, s, H-29), $4.65(1 \mathrm{H}, \mathrm{m},-\mathrm{NHCH}-), 3.90\left(2 \mathrm{H}, \mathrm{s}, H_{2}-30\right), 3.17(1 \mathrm{H}, \mathrm{dd}$, $J=9.7,5.4 \mathrm{~Hz}, H-3), 3.10-3.03(1 \mathrm{H}, \mathrm{m}, H-19), 1.00\left(6 \mathrm{H}, \mathrm{s}\right.$, leucine moiety $\left.-\left(\mathrm{CH}_{3}\right)_{2}\right), 0.96(6 \mathrm{H}, \mathrm{s}$, $\left.2 \times \mathrm{CH}_{3}\right), 0.83,0.80,0.79$ (3H each, s, $\left.3 \times \mathrm{CH}_{3}\right), \mathrm{MS} \mathrm{m} / 2$ 646.41, $648.39\left(\mathrm{M}^{-}-1\right)$; Compound 15: ${ }^{1} \mathrm{H}$ NMR $\left(300 \mathrm{MHz}, \mathrm{CDCl}_{3}\right): \delta 5.88(1 \mathrm{H}, \mathrm{d}, J=8.0 \mathrm{~Hz},-\mathrm{CONH}-), 4.93,4.92(2 \mathrm{H}, b r \mathrm{~s}, H-29)$, 4.63-4.58 (1H, m, -NHCH-), $3.90\left(2 \mathrm{H}, \mathrm{s}, \mathrm{H}_{2}-30\right), 3.47\left(2 \mathrm{H}, \mathrm{m}, 30-\mathrm{OCH}_{2} \mathrm{CH}_{3}\right), 3.18(1 \mathrm{H}, \mathrm{dd}, J=$ 11.1, $5.4 \mathrm{~Hz}, H-3), 2.99(1 \mathrm{H}, \mathrm{m}, H-19), 2.50-2.32(1 \mathrm{H}, \mathrm{m}, H-13), 1.00\left(9 \mathrm{H}, b r \mathrm{~s}, 30-\mathrm{OCH}_{2} \mathrm{CH}_{3}\right.$, leucine moiety - $\left.\left(\mathrm{CH}_{3}\right)_{2}\right), 0.96\left(6 \mathrm{H}, \mathrm{s}, 2 \times \mathrm{CH}_{3}\right), 0.89,0.86,0.85\left(3 \mathrm{H}\right.$ each, s, $\left.3 \times \mathrm{CH}_{3}\right), \mathrm{MS} \mathrm{m} / z$ $612.40\left(\mathrm{M}^{-}-1\right)$; Compound 16: ${ }^{1} \mathrm{H}$ NMR $\left(300 \mathrm{MHz}^{\mathrm{C}} \mathrm{CDCl}_{3}\right): \delta 6.13(1 \mathrm{H}, \mathrm{br} \mathrm{s},-\mathrm{CONH}-), 4.91$, $4.90(2 \mathrm{H}, b r \mathrm{~s}, H-29), 4.52(1 \mathrm{H}, \mathrm{m},-\mathrm{NHCH}-), 3.90\left(2 \mathrm{H}, \mathrm{s}, H_{2}-30\right), 3.36(2 \mathrm{H}, \mathrm{t}, J=6.9 \mathrm{~Hz}, 30-$ $\left.\mathrm{OCH}_{2} \mathrm{CH}_{2} \mathrm{CH}_{3}\right), 3.18(1 \mathrm{H}, \mathrm{dd}, J=11.1,5.4 \mathrm{~Hz}, \mathrm{H}-3), 2.99(1 \mathrm{H}, \mathrm{m}, \mathrm{H}-19), 0.96,0.94,0.92,0.89$ $\left(15 \mathrm{H}, \mathrm{m}, 30-\mathrm{O}\left(\mathrm{CH}_{2}\right)_{2} \mathrm{CH}_{3}\right.$, leucine moiety $\left.-\left(\mathrm{CH}_{3}\right)_{2}, \mathrm{CH}_{3}-23,24\right), 0.82,0.81,0.79$ (3H each, s, $3 \times$ $\left.\mathrm{CH}_{3}\right), \mathrm{MS} \mathrm{m} / z 626.50\left(\mathrm{M}^{-}-1\right)$; Compound 17: ${ }^{1} \mathrm{H} \mathrm{NMR}\left(300 \mathrm{MHz}, \mathrm{CDCl}_{3}\right): \delta$ 7.68-7.62 (2H, m, Ar- $\left.H-3^{\prime}\right), 7.28-7.20$ (3H, m, Ar- $\left.H-2^{\prime}, 4^{\prime}\right), 5.97$ (1H, br s, -CONH-), 4.91, 4.90 (2H, br s, H-29), $4.44(1 \mathrm{H}, \mathrm{m},-\mathrm{NHCH}-), 3.93\left(2 \mathrm{H}, \mathrm{s}, \mathrm{H}_{2}-30\right), 3.64\left(2 \mathrm{H}, \mathrm{t}, J=7.2 \mathrm{~Hz}, 30-\mathrm{OCH}_{2} \mathrm{CH}_{2} \mathrm{Ph}\right), 3.17(1 \mathrm{H}$, $\mathrm{dd}, J=11.1,5.4 \mathrm{~Hz}, H-3), 2.91(1 \mathrm{H}, \mathrm{m}, H-19), 2.57\left(2 \mathrm{H}, \mathrm{m}, 30-\mathrm{OCH}_{2} \mathrm{CH}_{2} \mathrm{Ph}\right), 0.95(12 \mathrm{H}, \mathrm{s}$, leucine moiety - $\left.\left(\mathrm{CH}_{3}\right)_{2}, \mathrm{CH}_{3}-23,24\right), 0.89,0.78,0.74$ (3H each, s, $\left.3 \times \mathrm{CH}_{3}\right), \mathrm{MS} \mathrm{m} / z 688.41\left(\mathrm{M}^{-}\right.$ - 1); Compound 18: ${ }^{1} \mathrm{H}$ NMR (300 MHz, $\left.\mathrm{CDCl}_{3}\right): \delta 7.27,7.16-7.13,6.85-6.82\left(4 \mathrm{H}, \mathrm{m}, \mathrm{Ar}-\mathrm{H}-2^{\prime}\right.$, 3'), 5.97 (1H, $b r$ s, -CONH-), 4.91, 4.89 (H each, $b r$ s, $H-29), 4.48$ (1H, m, -NHCH-), 3.93 (2H, s, $\left.\mathrm{H}_{2}-30\right), 3.79\left(3 \mathrm{H}, \mathrm{s}, \mathrm{Ar}-\mathrm{OCH} H_{3}\right), 3.60\left(2 \mathrm{H}, \mathrm{t}, J=7.2 \mathrm{~Hz}, 30-\mathrm{OCH}_{2} \mathrm{CH}_{2} \mathrm{Ph}\left(p-\mathrm{OCH}_{3}\right)\right), 3.17(1 \mathrm{H}, \mathrm{dd}$, 
$J=11.1,5.4 \mathrm{~Hz}, H-3), 2.85(1 \mathrm{H}, \mathrm{t}, J=7.5 \mathrm{~Hz}, H-19), 2.39\left(3 \mathrm{H}, \mathrm{m}, 30-\mathrm{OCH}_{2} \mathrm{CH}_{2} \mathrm{Ph}\left(p-\mathrm{OCH}_{3}\right)\right.$, $H$-13), 0.95, 0.93, $0.90\left(15 \mathrm{H}, \mathrm{s}\right.$, leucine moiety $\left.-\left(\mathrm{CH}_{3}\right)_{2}, 3 \times \mathrm{CH}_{3}\right), 0.79,0.75$ (3H each, s, $2 \times$ $\left.\mathrm{CH}_{3}\right), \mathrm{MS} \mathrm{m} / z, 718.50\left(\mathrm{M}^{-}-1\right)$; Compound 19: ${ }^{1} \mathrm{H} \mathrm{NMR}\left(300 \mathrm{MHz}, \mathrm{CDCl}_{3}\right): \delta 7.16-6.80(4 \mathrm{H}, \mathrm{m}$, Ar- $\left.H-2^{\prime}, 3^{\prime}\right), 5.96$ (1H, br s, -CONH-), 4.90, 4.89 (2H, br s, H-29), 4.48 (1H, m, -NHCH-), 3.92 $\left(2 \mathrm{H}, \mathrm{s}, H_{2}-30\right), 3.61\left(2 \mathrm{H}, \mathrm{t}, J=7.2 \mathrm{~Hz}, 30-\mathrm{OCH}_{2} \mathrm{CH}_{2} \mathrm{Ph}(p-\mathrm{F})\right), 3.17(1 \mathrm{H}, \mathrm{m}, H-3), 2.87(1 \mathrm{H}, \mathrm{t}, J=$ $7.5 \mathrm{~Hz}, \mathrm{H}-19), 2.36-2.10\left(3 \mathrm{H}, \mathrm{m}, 30-\mathrm{OCH}_{2} \mathrm{CH}_{2} \mathrm{Ph}(p-\mathrm{F}), H-13\right), 0.95,0.88$ (15H, s, leucine moiety $\left.-\left(\mathrm{CH}_{3}\right)_{2}, 3 \times \mathrm{CH}_{3}\right), 0.75,0.73\left(3 \mathrm{H}\right.$ each, s, $\left.2 \times \mathrm{CH}_{3}\right), \mathrm{MS} \mathrm{m} / z .706 .40\left(\mathrm{M}^{-}-1\right) ;$ Compound $20:{ }^{1} \mathrm{H}$ NMR (300 MHz, $\left.\mathrm{CDCl}_{3}\right): \delta$ 7.56-7.28 (4H, m, Ar- $\left.H-2^{\prime}, 3^{\prime}\right), 5.96(1 \mathrm{H}, b r \mathrm{~s},-\mathrm{CONH}-), 4.91,4.90$ $(2 \mathrm{H}, b r \mathrm{~s}, H-29), 4.48(1 \mathrm{H}, \mathrm{m},-\mathrm{NHCH}-), 3.91\left(2 \mathrm{H}, \mathrm{s}, H_{2}-30\right), 3.60(2 \mathrm{H}, \mathrm{t}, J=7.0 \mathrm{~Hz}, 30-$

$\left.\mathrm{OCH}_{2} \mathrm{CH}_{2} \mathrm{Ph}(p-\mathrm{Br})\right), 3.17(1 \mathrm{H}, \mathrm{dd}, J=11.0,5.6 \mathrm{~Hz}, H-3), 2.89(1 \mathrm{H}, \mathrm{t}, J=7.5 \mathrm{~Hz}, H-19), 2.39$ $\left(1 \mathrm{H}, \mathrm{m}, 30-\mathrm{OCH}_{2} \mathrm{CH}_{2} \mathrm{Ph}(\mathrm{p}-\mathrm{Br})\right), 0.96\left(12 \mathrm{H}\right.$, s, leucine moiety $\left.-\left(\mathrm{CH}_{3}\right)_{2}, 2 \times \mathrm{CH}_{3}\right), 0.82,0.79,0.75$ (3H each, s, $\left.3 \times \mathrm{CH}_{3}\right)$, MS m/z $766.38\left(\mathrm{M}^{-}-1\right)$; Compound 21: ${ }^{1} \mathrm{H} \mathrm{NMR}\left(300 \mathrm{MHz}, \mathrm{CDCl}_{3}\right): \delta$ 7.18-6.87 (4H, m, Ar- H-2', 3'), $5.96(1 \mathrm{H}$, br s, -CONH-), 4.91, $4.90(2 \mathrm{H}$, br s, $H-29), 4.48(1 \mathrm{H}, \mathrm{m}$, -NHCH-), $3.91\left(2 \mathrm{H}, \mathrm{s}, \mathrm{H}_{2}-30\right), 3.62\left(2 \mathrm{H}, \mathrm{t}, J=6.8 \mathrm{~Hz}, 30-\mathrm{OCH}_{2} \mathrm{CH}_{2} \mathrm{Ph}(p-\mathrm{Cl})\right), 3.17(1 \mathrm{H}, \mathrm{dd}, J=$ 11.0, 5.6 Hz, H-3), $2.87(1 \mathrm{H}, \mathrm{t}, J=7.5 \mathrm{~Hz}, H-19), 2.36-2.06\left(3 \mathrm{H}, \mathrm{m}, 30-\mathrm{OCH}_{2} \mathrm{CH}_{2} \mathrm{Ph}(p-\mathrm{Cl})\right.$, $H$-13), $0.96\left(15 \mathrm{H}\right.$, s, leucine moiety $\left.-\left(\mathrm{CH}_{3}\right)_{2}, 3 \times \mathrm{CH}_{3}\right), 0.81,0.76\left(3 \mathrm{H} \mathrm{each}, \mathrm{s}, 2 \times \mathrm{CH}_{3}\right), \mathrm{MS} \mathrm{m} / \mathrm{z}$ $722.40\left(\mathrm{M}^{-}-1\right)$; Compound 22: ${ }^{1} \mathrm{H} \mathrm{NMR}\left(300 \mathrm{MHz}, \mathrm{CDCl}_{3}\right): \delta 5.61(1 \mathrm{H}, \mathrm{d}, J=8 \mathrm{~Hz},-\mathrm{CONH}-)$, 4.92, 4.90 (H each, s, H-29), $4.59(1 \mathrm{H}, \mathrm{m},-\mathrm{NHCH}-), 3.94\left(2 \mathrm{H}, \mathrm{s}, H_{2}-30\right), 3.72(4 \mathrm{H}, \mathrm{m},-$

$\left.\mathrm{N}\left(\mathrm{CH} 2 \mathrm{CH}_{2}\right)_{2} \mathrm{O}\right), 3.58\left(2 \mathrm{H}, \mathrm{t}, J=5.7 \mathrm{~Hz}, 30-\mathrm{OCH}_{2} \mathrm{CH}_{2}\right.$-morpholine $), 3.18(1 \mathrm{H}, \mathrm{dd}, J=11.4,4.6$ $\mathrm{Hz}, H-3), 3.01(1 \mathrm{H}, \mathrm{m}, H-19), 2.60\left(2 \mathrm{H}, \mathrm{t}, J=5.4 \mathrm{~Hz}, 30-\mathrm{OCH}_{2} \mathrm{CH}_{2}\right.$-morpholine), $2.53(4 \mathrm{H}, \mathrm{m},-$ $\left.\mathrm{N}\left(\mathrm{CH}_{2} \mathrm{CH}_{2}\right)_{2} \mathrm{O}\right), 1.00\left(6 \mathrm{H}, \mathrm{s}\right.$, leucine moiety $\left.-\left(\mathrm{CH}_{3}\right)_{2}\right), 0.96\left(6 \mathrm{H}, \mathrm{s}, 2 \times \mathrm{CH}_{3}\right), 0.89,0.85,0.80(3 \mathrm{H}$ each, $\left.\mathrm{s}, 3 \times \mathrm{CH}_{3}\right)$, MS $\mathrm{m} / 2697.40\left(\mathrm{M}^{-}-1\right)$.

23. 20S proteasome assay: $20 \mathrm{~S}$ proteasome assay kits were purchased from Calbiochem, San Diego, CA. Measurement of the hydrolysis of the fluorogenic substrate Suc-Leu-Leu-Val-Tyr-AMC in the presence of the proteasome activator PA28 $(16 \mu \mathrm{g} / \mathrm{mL})$ is used to detect the chymotrypsin-like activity of the human $20 \mathrm{~S}$ proteasome. Fluorescence generated from the proteolytic reaction in the presence of various concentrations of BA derivatives was measured using a BioTek fluorometer (Winooski, Vermont). The $50 \%$ inhibitory concentration $\left(\mathrm{IC}_{50}\right)$ is defined as the inhibitor concentration that reduces the reaction rate by $50 \%$. The velocity of reaction ( $\triangle \mathrm{RFU}(360 / 460) /$ min) was plotted against the log-concentration of the inhibitor to determine the $\mathrm{IC}_{50}$. 


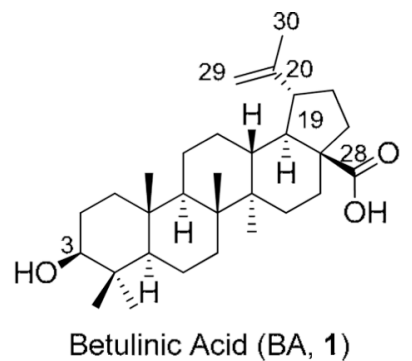

Betulinic Acid (BA, 1)

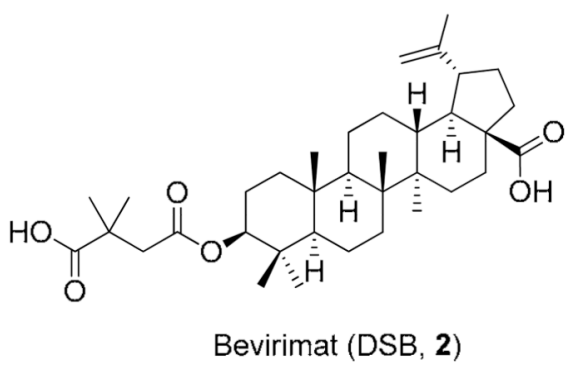

Bevirimat (DSB, 2)

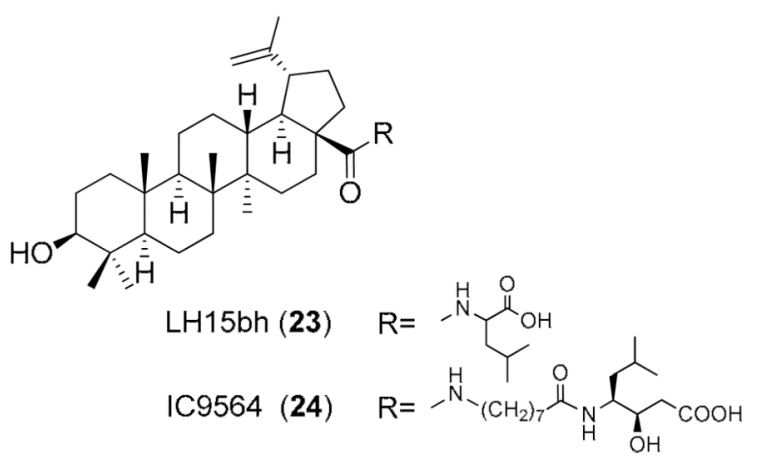

Figure 1.

Structures of BA (1) and its derivatives $(\mathbf{2}, \mathbf{2 3}, \mathbf{2 4})$. 


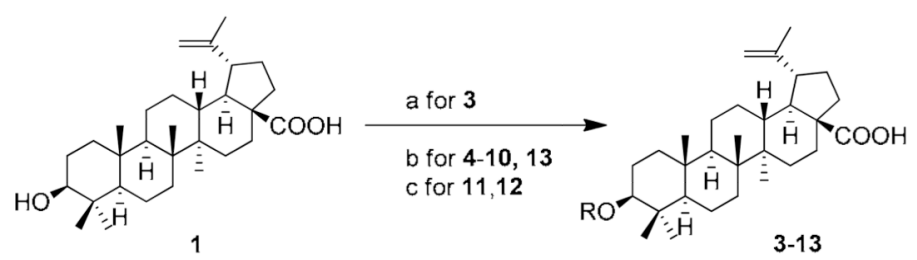

1

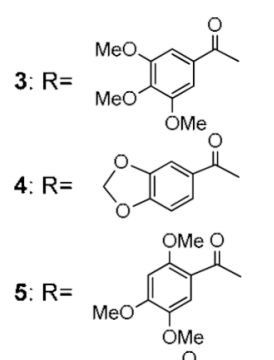

$7: \mathrm{R}=\mathrm{MeO}^{\mathrm{MeO}}$<smiles>[R]=[B]</smiles>

9: $R=$

6: $R=$<smiles>CC(=O)c1ccccc1</smiles>

11: $\mathrm{R}=\mathrm{HO}_{\longrightarrow}$<smiles></smiles>

13: $R={ }_{0}^{H O}$

Scheme 1.

Reactions and conditions: (a) acid chloride, $\mathrm{Et}_{3} \mathrm{~N}, \mathrm{CH}_{2} \mathrm{Cl}_{2}$, rt; (b) acid, EDCI, DMAP, $\mathrm{Et}_{3} \mathrm{~N}$, $\mathrm{CH}_{2} \mathrm{Cl}_{2}$, rt; (c) acid anhydride, DMAP, pyridine, reflux. 

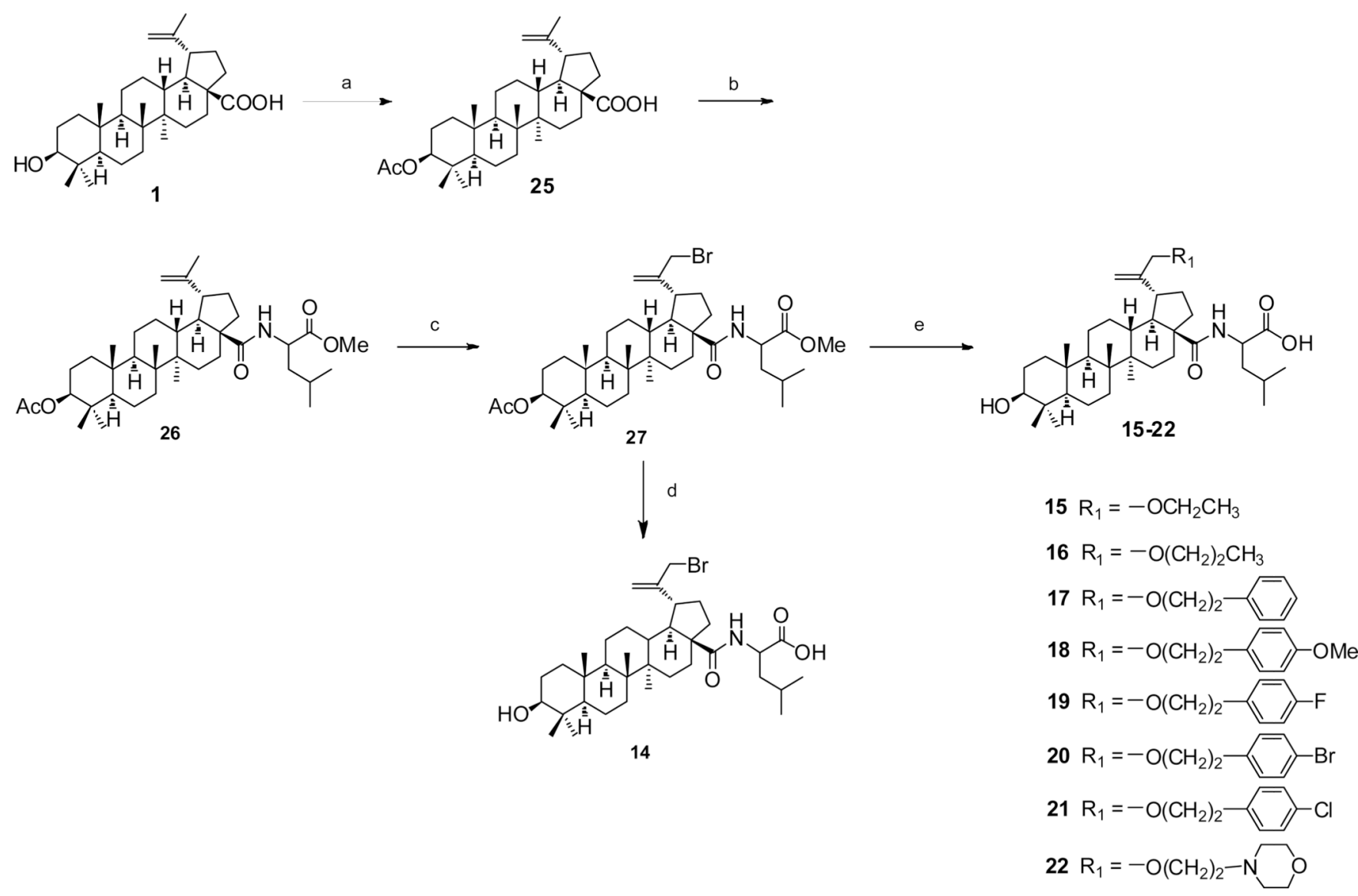

Scheme 2.

Reactions and conditions: (a) $\mathrm{Ac}_{2} \mathrm{O}$, pyridine, $78^{\circ} \mathrm{C}$, $4 \mathrm{~h}$; (b) $\left(\mathrm{CO}_{2}\right) \mathrm{Cl}_{2}$, leucine methyl ester, $\mathrm{Et}_{3} \mathrm{~N}, \mathrm{CH}_{2} \mathrm{Cl}_{2}$, rt; (c) NBS, acetonitrile, rt; (d) $2 \mathrm{~N} \mathrm{NaOH}, \mathrm{MeOH} / \mathrm{THF}$, rt; (e) step 1: nucleophilic compounds, NaH, THF; step 2: $\mathrm{MeOH}-\mathrm{H}_{2} \mathrm{O}$. 
Table 1

The inhibition on the chymotrypsin-like activity of 20S proteasome of 3-24 with $\mathbf{2}$, LLM-F, and lactacystin as controls.

\begin{tabular}{lclc}
\hline Compound & $\mathbf{I C}_{\mathbf{5 0}}(\boldsymbol{\mu M}) \boldsymbol{a}$ & Compound & $\mathbf{I C}_{\mathbf{5 0}}(\boldsymbol{\mu M})$ \\
\hline $\mathbf{3}$ & $>40$ & $\mathbf{1 6}$ & 3.67 \\
$\mathbf{4}$ & $>40$ & $\mathbf{1 7}$ & 2.47 \\
$\mathbf{5}$ & 10.91 & $\mathbf{1 8}$ & 3.34 \\
$\mathbf{6}$ & 11.06 & $\mathbf{1 9}$ & 2.40 \\
$\mathbf{7}$ & $>40$ & $\mathbf{2 0}$ & 1.56 \\
$\mathbf{8}$ & 7.99 & $\mathbf{2 1}$ & 1.80 \\
$\mathbf{9}$ & $>40$ & $\mathbf{2 2}$ & 3.13 \\
$\mathbf{1 0}$ & 7.05 & $\mathbf{2 3}$ & $>40$ \\
$\mathbf{1 1}$ & 2.96 & $\mathbf{2 4}$ & $>40$ \\
$\mathbf{1 2}$ & $>40$ & $\mathbf{2}$ & 9.20 \\
$\mathbf{1 3}$ & 1.42 & LLM-F & 5.25 \\
$\mathbf{1 4}$ & 5.85 & lactacystin & 5.60 \\
$\mathbf{1 5}$ & 13.21 & & \\
\hline
\end{tabular}

${ }^{a}$ The IC50 values in the table are the averages from three experiments. 